\title{
Engineered T cells targeting E7 mediate regression of human papillomavirus cancers in a murine model
}

\author{
Benjamin Y. Jin, ${ }^{1}$ Tracy E. Campbell, ${ }^{1}$ Lindsey M. Draper, ${ }^{1}$ Sanja Stevanović, ${ }^{1}$ Bianca Weissbrich, ${ }^{2}$ \\ Zhiya Yu, ${ }^{3}$ Nicholas P. Restifo, ${ }^{3}$ Steven A. Rosenberg, ${ }^{3}$ Cornelia L. Trimble, ${ }^{4}$ \\ and Christian S. Hinrichs ${ }^{1}$ \\ 'Experimental Transplantation and Immunology Branch, National Cancer Institute, NIH, Bethesda, Maryland, USA. \\ ${ }^{2}$ Kite Pharma, Amsterdam, Netherlands. ${ }^{3}$ Surgery Branch, National Cancer Institute, NIH, Bethesda, Maryland, USA. \\ ${ }^{4}$ Johns Hopkins University School of Medicine, Baltimore, Maryland, USA.
}

T cell receptor (TCR) T cell therapy is a promising cancer treatment modality. However, its successful development for epithelial cancers may depend on the identification of high-avidity TCRs directed against tumor-restricted target antigens. The human papillomavirus (HPV) E7 antigen is an attractive therapeutic target that is constitutively expressed by $\mathrm{HPV}^{+}$cancers but not by healthy tissues. It is unknown if genetically engineered TCR T cells that target E7 can mediate regression of $\mathrm{HPV}^{+}$cancers. We identified an HPV-16 E7-specific, HLA-A*02:01-restricted TCR from a uterine cervix biopsy from a woman with cervical intraepithelial neoplasia. This TCR demonstrated high functional avidity, with CD8 coreceptor-independent tumor targeting. Human T cells transduced to express the TCR specifically recognized and killed HPV-16+ cervical and oropharyngeal cancer cell lines and mediated regression of established HPV-16+ human cervical cancer tumors in a mouse model. These findings support the therapeutic potential of this approach and established the basis for an E7 TCR gene therapy clinical trial in patients with metastatic HPV+ cancers (NCT02858310).

Authorship note: BY] and TEC contributed equally to this work.

Conflict of interest: CSH and SAR are inventors on $\mathrm{NIH}$ patents related to the E6 TCR, the E7 TCR, and TIL therapy for HPV-associated cancers (patent application no. 62/004,335, $61 / 846,167,61 / 846,161)$. Both of their NIH laboratories receive research funding through cooperative research and development agreements with Kite Pharma.

Submitted: December 27, 2017

Accepted: March 14, 2018

Published: April १९, 2018

\section{Reference information:}

JCI Insight. 2018;3(8):e99488. https://doi.org/10.1172/jci. insight. 99488.

\section{Introduction}

Human papillomavirus (HPV) causes cancers of the uterine cervix, oropharynx, anus, vulva, vagina, and penis (1-3). These malignancies are difficult to treat and metastatic disease is generally incurable (4-6). It is hoped that in the future HPV-associated cancers will be prevented with vaccines and cancer screening, but at the present time they cause more than 7,000 deaths in the United States and hundreds of thousands of deaths around the world each year $(7,8)$. Innovative new treatment strategies are urgently needed. Antigen receptor $\mathrm{T}$ cell therapy is a novel immunotherapeutic approach that may represent a breakthrough for the treatment of certain cancers $(9,10)$. It is based on the administration of $\mathrm{T}$ cells that are genetically engineered to express a chimeric antigen receptor (CAR) or T cell receptor (TCR) that targets a tumor antigen $(11,12)$. CAR T cells directed against CD19 have demonstrated remarkable clinical activity in B cell malignancies (13-16). Tumor responses have also been reported for TCR T cells targeting cancer/testis antigen 1 in melanoma and synovial cell sarcoma (17). However, clinical development of antigen receptor T cell therapy for other solid tumors has been limited in large part by on-target, off-tumor toxicities (18-20). Advances in the treatment of solid tumors may depend on the discovery and development of receptors that target antigens that are consistently and highly expressed by tumors but are not expressed by healthy tissues (21).

The high-risk HPV E6 and E7 oncoproteins are viral antigens that are constitutively expressed by HPV-associated cancers but not expressed by healthy tissues (1). They contribute not only to malignant transformation but also to survival of transformed cells, making them particularly attractive therapeutic targets $(9,22,23)$. However, there are conflicting rationale to support or refute whether these antigens might be effectively targeted with cancer immunotherapy. T cell targeting of tumor cells that express these antigens may be undermined by complex mechanisms of evasion, including antigen "disguise" by sequence similarity with human proteins (24), interference with antigen processing and presentation (25), dysregulation of cytokine and chemokine pathways $(26,27)$, and a hostile tumor microenvironment $(28,29)$. T cells that are specific for the HPV oncoproteins frequently infiltrate HPV-associated cancers but nonetheless fail 
to control the disease $(30,31)$. In addition, oncoprotein-directed vaccine therapy has been unsuccessful in patients with metastatic $\mathrm{HPV}^{+}$cancers (32-35). Despite these findings, some data suggest that $\mathrm{HPV}^{+}$cancers may be targetable with $\mathrm{T}$ cell therapy $(36,37)$. Adoptive transfer of tumor-infiltrating $\mathrm{T}$ cells (TILs) can induce complete regression of metastatic cervical cancer $(36,37)$. In addition, $\mathrm{T}$ cells that target E6 with a high-avidity TCR can recognize and kill HPV-16+ tumor cell lines in vitro (38). We sought to identify a TCR that specifically targeted HPV-16 E7 and to determine if T cells genetically engineered to express this TCR could overcome the evasive forces of human $\mathrm{HPV}^{+}$tumors to engage and kill tumor cell lines in vitro and to mediate regression of established tumors in vivo.

HPV-16 is the most common oncogenic HPV type in humans. It accounts for approximately 65, 70, and 90 percent of cervical, oropharyngeal, and anal cancers, respectively (39). HLA-A*02:01 is the most common class I allele in the United States, expressed in approximately $40-50$ percent of people of European descent (40). HPV-16 E7 may be a more desirable target than HPV-16 E6, as it demonstrates less sequence variation and it harbors an epitope with greater HLA-A*02:01-binding affinity than any known epitope from E6 (41). In prior research, we had screened TILs from HLA-A*02:01+ patients with metastatic HPV- $16^{+}$cancers and failed to identify an HLA-A*02:01-restricted, E7-specific TCR. We speculated that patients who develop advanced $\mathrm{HPV}^{+}$cancers may possess inherently weak TIL responses against the HPV-16 oncoproteins and that samples from these patients may not be optimal for discovery of HPV-specific TCRs. Therefore, we turned our attention instead to the study of cervix-infiltrating lymphocytes (CILs) from patients with HPV-16 ${ }^{+}$cervical intraepithelial neoplasia II/III.

\section{Results}

Discovery of a high-avidity HLA-A*02:01-restricted TCR that targets HPV-16 E7. Cryopreserved CILs from 10 patients were expanded and tested for HPV-16 E6 or E7 reactivity with an IFN- $\gamma$ production assay. CILs from a single patient (patient 5048) demonstrated specific recognition of E7 (Figure 1A). Patient 5048 was determined by the NIH Clinical Center HLA Laboratory to express the HLA-A*02:01 allele. The E7 ${ }_{11-19}$ epitope of $\mathrm{E} 7$ has been reported to be naturally processed and presented by the HLA-A*02:01 molecule based on study of tumor cells with peptide elution and mass spectroscopy (41). To determine if a population of $\mathrm{T}$ cells in CILs from patient 5048 (5048 CILs) targeted the HLA-A*02:01-E7 ${ }_{11-19}$ peptide-major histocompatibility (p-MHC) complex, we analyzed $5048 \mathrm{CIL}$ binding to fluorescently labeled HLA-A*02:01-E7 ${ }_{11-19}$ tetramers by flow cytometry. A CD8 ${ }^{+} \mathrm{T}$ cell population that comprised 5 percent of 5048 CILs showed tetramer binding (Figure 1B). A T cell clone with high tetramer binding was isolated, and the TCR $\alpha$ chain and $\beta$ chain nucleotide sequences were determined as described in the Methods. An MSGV1 retroviral vector for expression of the TCR chains was constructed. The TCR constant regions were exchanged for their mouse counterparts, which generally improves pairing of the $\alpha$ and $\beta$ chains $(42,43)$. The initial vector was designed with the $\alpha$ chain, followed by a furin-P2A linker, followed by the $\beta$ chain. T cells that were transduced with this vector displayed expression of the TCR $\beta$ chain (Figure 1C). However, about half of the $\beta$ chain-expressing T cells failed to bind HLA-A*02:01-E7 ${ }_{11-19}$ tetramers (Figure 1C).

We speculated that in some T cells the introduced TCR $\alpha$ and $\beta$ chains were not pairing properly to form functional receptors with HLA-A*02:01-E7 ${ }_{11-19}$ specificity. We tested if tetramer binding was improved by (a) reversal of the $\alpha$ and $\beta$ chain order, (b) addition of an interchain disulfide bond (44), or (c) hydrophobic substitutions to the $\alpha$ chain transmembrane region (45) (Figure 1C). Each of these modifications increased the frequency of TCR $\beta$ chain-expressing T cells that bound to $\mathrm{p}-\mathrm{MHC}$ tetramers (Figure $1 \mathrm{C}$ ). We selected the construct with the $\beta-\alpha$ chain order, added disulfide bond, and hydrophobic substitutions (referred to herein as the E7 TCR) as our lead candidate for further testing, as this construct showed the lowest frequency of transduced T cells (TCR $\beta$ chain ${ }^{+}$) that did not bind tetramer (tetramer) (Figure 1, C and D).

E7 TCR T cells specifically recognize and kill HLA- $A^{*} 02: 01^{+} H P V-16^{+}$tumor cells. We next performed experiments to confirm the restriction element and target antigen of the E7 TCR. Recognition of the tumor cells lines CaSki, 624-E7, and SCC152 (HLA-A*02:01+ and E7+ lines) by E7 TCR T cells, as measured by IFN- $\gamma$ production, was inhibited by antibody blockade of HLA class I but not HLA class II molecules, which indicated that target recognition was class I restricted (Figure 2A). The target antigen and restriction element were more specifically interrogated in an experiment with 293 cells as targets. These target cells were recognized by E7 TCR T cells only if they expressed both the HLA-A*02:01 molecule and either the $\mathrm{E}_{11-19}$ peptide or the full-length E7 protein (Figure $2 \mathrm{~B}$ ). These data indicated that the E7 TCR recognized the $\mathrm{E} 7_{11-19}$ epitope in an HLA-A*02:01-restricted fashion. 


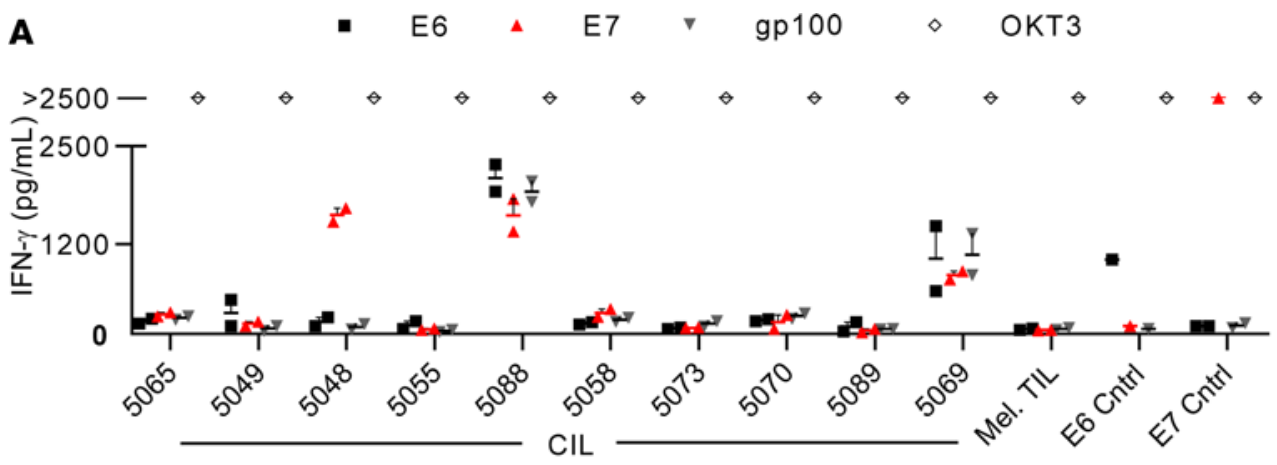

B
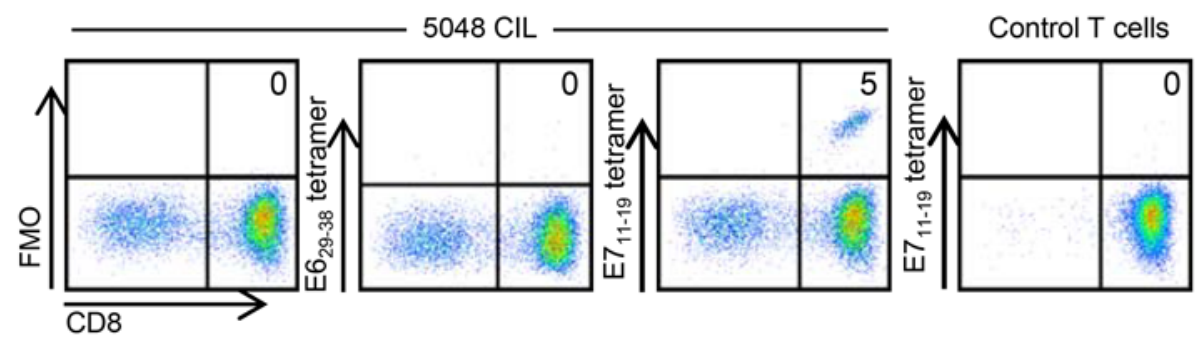

C

Wild-type
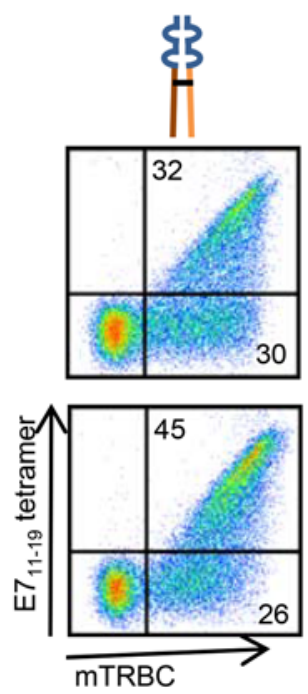

D

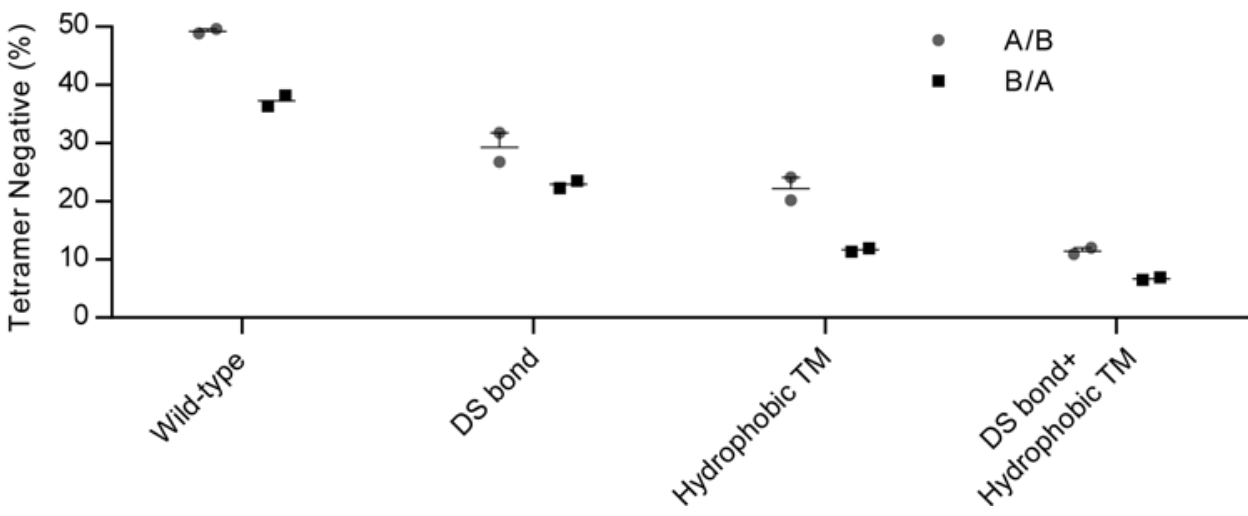

Figure 1. Identification and optimized expression of a TCR that targets HPV-16 E7. (A) IFN- $\gamma$ production assay testing CILs from 10 patients for recognition of HPV-16 E6 or E7. CILs from each patient were cocultured with autologous dendritic cells loaded with peptide pools spanning the indicated antigen. gp100, also known as melanocyte protein $\mathrm{PMEL}$, is the negative control protein. OKT3 is a positive control of T cells stimulated with plate-bound anti-CD3 antibody. Mel TILs are a negative control of TILs from a melanoma patient. E6 Cntrl and E7 Cntrl are positive controls of $T$ cells genetically engineered to express an E6- or E7-targeting TCR, respectively. The concentration of IFN- $\gamma$ in supernatants after overnight coculture is displayed. (B) Flow cytometry analysis of 5048 CILs binding to $\mathrm{E7}_{11-19}-\mathrm{HLA}-\mathrm{A}^{*} 02: 01$ tetramers. $\mathrm{EG}_{29-38}$ tetramer is a negative control tetramer. Control $T$ cells are activated, third-party $T$ cells. Dot plots are gated on live lymphocytes. The tetramers were PE labeled. The anti-CD8 antibody was PE-Cy7 labeled. FMO, fluorescence minus one. (C) Flow cytometry analysis of $\mathrm{T}$ cells transduced to express the E7 TCR. The schematic for each construct is shown above each dot plot. Orange and brown indicate $\alpha$ and $\beta$ chain constant regions, respectively. The wild-type interchain disulfide bond is black. The introduced disulfide bond is red. Hydrophobic substitutions to the $\alpha$ chain transmembrane region are green. Variable regions are blue. The $\alpha$ and $\beta$ chain gene order in the vector insert is indicated to the right of each row. Dot plots are gated on live lymphocytes. Quadrant frequencies are indicated. DS, disulfide; TM, transmembrane; mTRBC, mouse TCR $\beta$ constant region. The tetramer is APC labeled. The anti-mTRBC antibody is PE labeled. (D) Data from the experiment in $\mathbf{C}$ are graphed to display the frequency of transduced T cells (mouse TCR $\beta$ chain-positive) that did not bind E7 11-19 $-\mathrm{HLA}-\mathrm{A}^{*} 02: 01$ tetramers. Error bars represent the SEM for technical replicates. For $\mathbf{C}$ and $\mathbf{D}$, the results are representative of 2 independent experiments. 
The ability of E7 TCR T cells to specifically recognize and mediate effector functions in response to HPV-16 ${ }^{+}$tumor cell lines was evaluated with cytokine production and T cell cytotoxicity assays. E7 TCR T cells showed production of IFN- $\gamma$ (Figure 2C) and TNF- $\alpha$ (Figure 2D) in response to each of the HLA-A*02:01+ HPV- $16^{+}$tumor cell lines tested. The cell lines that were recognized included CaSki (a cervical cancer cell line that has been previously reported to evade T cell recognition through defects in MHC complex class I, transporter proteins associated with antigen-processing molecules, and proteasome subunits) (28) and SCC 90 and SCC152 (two head and neck cancer cell lines). Tumor lines that lacked either the HLA-A*02:01 restriction element or the E7 target antigen were not recognized (Figure 2, C and D). The ability of E7 TCR T cells to kill tumor cells was assessed with a real-time impedance-based cytolysis assay (46) (Figure 2E). Each of the target cell lines that expressed HLA-A*02:01 and E7 were killed at a low effector-to-target ratio, including two cervical cancer cell lines (4050 and CaSki) and two head and neck cancer lines (SCC90 and SCC152) (Figure 2E, top). Tumor cell lines that did not express the HLA-A*02:01 molecule or the HPV-16 E7 molecule were not killed (Figure 2E, bottom). These results demonstrated that E7 TCR T cells could specifically engage and mediate T cell effector functions against HPV-16 tumor lines.

E7 TCR T cell cross-reactivity against epitopes of human proteins was weak to absent. The human TCR repertoire demonstrates inherent cross-reactivity that permits an estimated $10^{8}$ unique TCRs to provide recognition of greater than $10^{15}$ potential peptides. In certain TCR gene-engineered T cell clinical trials, TCR cross-reactivity has resulted in unintended targeting of healthy human tissues and severe toxicities (18, 20, 47). To assess E7 TCR T cells for cross-reactivity, we first identified by alanine scanning the residues of the E7 ${ }_{11-19}$ peptide that mediate recognition of that peptide by the E7 TCR (Figure 3A). Alanine substitutions at positions $2,4,5,6$, and 7 reduced the recognition of $\mathrm{E}_{11-19}$ by the $\mathrm{E} 7 \mathrm{TCR}$, which suggested that those amino acid residues contribute to the E7 TCR recognition of a target peptide. A BLAST search was performed to identify human peptides that share $\mathrm{E}_{11-19}$ residues at positions 4-7 and that possess an HLA-A*02:01 anchor-binding residue at the anchor position 2 (L, M, T, or A) and the anchor position 9 (V, I, L, T, or A) (48). Six human peptides were identified. No reactivity against these peptides was detected (Figure 3B). To expand the cross-reactivity testing, a BLAST search was conducted to identify human peptides with similar sequences. A cutoff of either 6 residues identical to E $7_{11-19}$ or 5 residues identical plus 1 conservative replacement was used. Nineteen candidate peptides were found. An initial screen for E7 TCR $\mathrm{T}$ cell reactivity against these peptides was performed using a high concentration of peptide $(1 \mu \mathrm{g} / \mathrm{ml}) \mathrm{load}$ ed onto T2 cells (Figure 3C). Six of the nineteen peptides displayed possible weak recognition. A second assay was performed using target cells that were loaded with titrated concentrations of peptide (Figure 3D). Targets with peptides at less than $100 \mathrm{ng} / \mathrm{ml}$ (still far above physiological levels) were not recognized, while recognition of the $\mathrm{E}_{11-19}$ peptide remained strong. These findings indicated weak to absent cross-reactivity of the E7 TCR against the epitopes of human peptides that were identified.

The E7 TCR displays high functional avidity. We assessed the functional avidity and the p-MHC monomer dissociation rate constant ( $K_{\text {off }}$ rate) for the E7 TCR. Comparison was made to a previously described TCR that targets an HLA-A*02:01-restricted epitope of HPV-16 E6 (38). In a functional avidity assay, E7 TCR T cells recognized cognate peptide at lower concentrations than E6 TCR T cells $(P<0.001)$ (Figure 4A). E7 TCR T cells demonstrated IFN- $\gamma$ release against cognate peptide at concentrations as low as 10 picomoles, whereas E6 TCR T cells demonstrated IFN- $\gamma$ release against cognate peptide at concentrations as low as 100 picomoles. As a second measure of avidity, the p-MHC monomer $K_{\text {off }}$ rate for each TCR was determined $(49,50)$ (Figure $4, \mathrm{~B}$ and C). The $t_{1 / 2}$ for the E7 TCR was 254 seconds and that for the E6 TCR was 49 seconds (E7 versus E6, $P<0.001$ ) (Figure $4 \mathrm{C}$ ). These findings indicated that the E7 TCR T cells possessed higher avidity for cognate peptide than E6 TCR T cells.

The ability of the E6 or E7 TCRs to mediate T cell engagement with and effector function against tumor cells was compared in functional assays in which either CD4 T cells or CD8 T cells were transduced to express each TCR. In CD4 T cells, the E7 TCR mediated greater IFN- $\gamma$ production against all target cells that expressed cognate antigen (CaSki $[P<0.0001]$, $4050[P<0.0001]$, and SCC152 $[P=0.0121]$ ) (Figure 4D). In CD8 T cells, the E7 TCR mediated significantly greater IFN- $\gamma$ production against CaSki cells $(P=$ 0.0001) but not against the other target cell lines (Figure 4E). E6 and E7 TCR-mediated killing of tumor cell lines was assessed by a real-time impedance-based cytolysis assay. The E6 TCR and the E7 TCR mediated CD4 and CD8 T cell killing of the 4050 tumor cell line. Neither receptor mediated killing of CaSki cells by CD4 T cells, and only the E7 TCR mediated killing of CaSki cells by CD8 T cells (Figure 4F). Overall, these findings indicated that the E7 TCR mediated equal or greater tumor recognition and T cell effector 
A

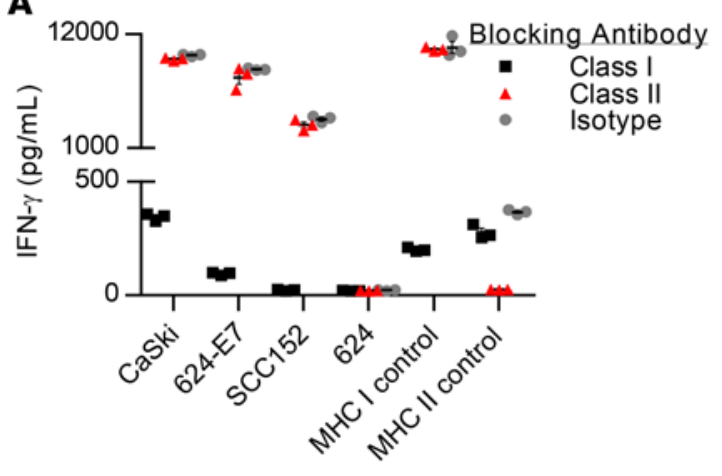

C

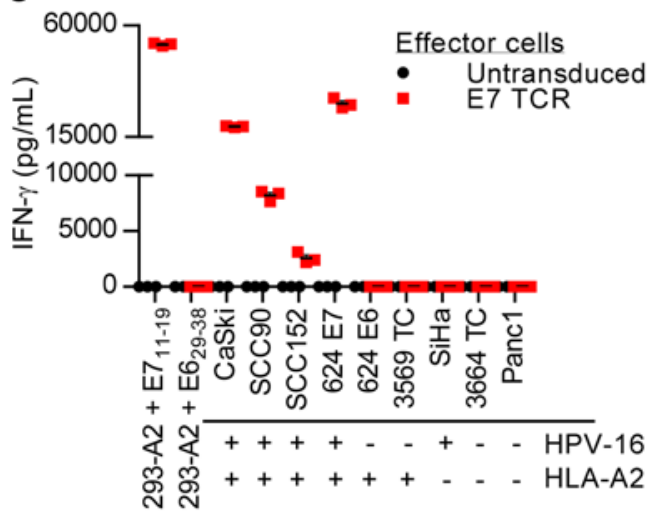

B

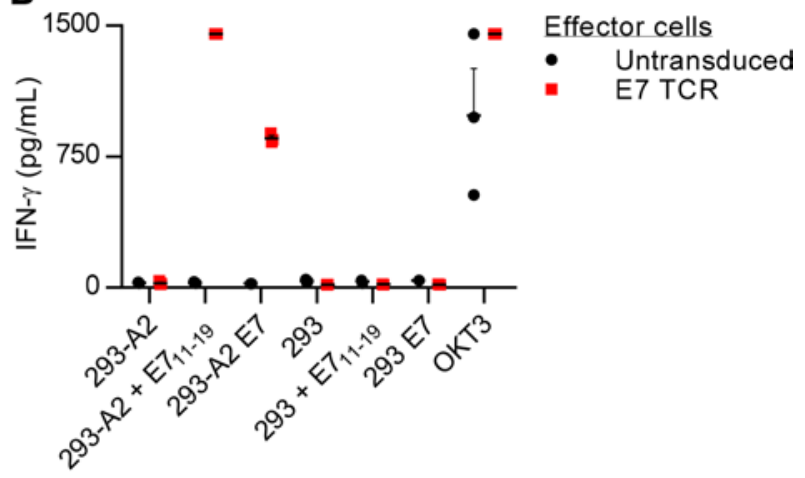

D

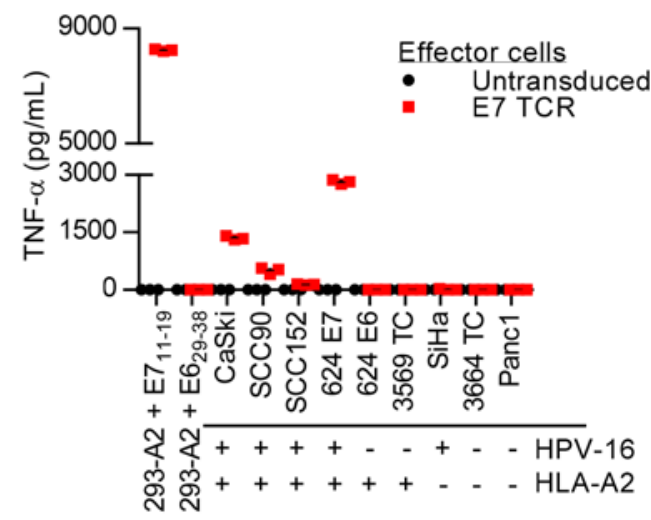

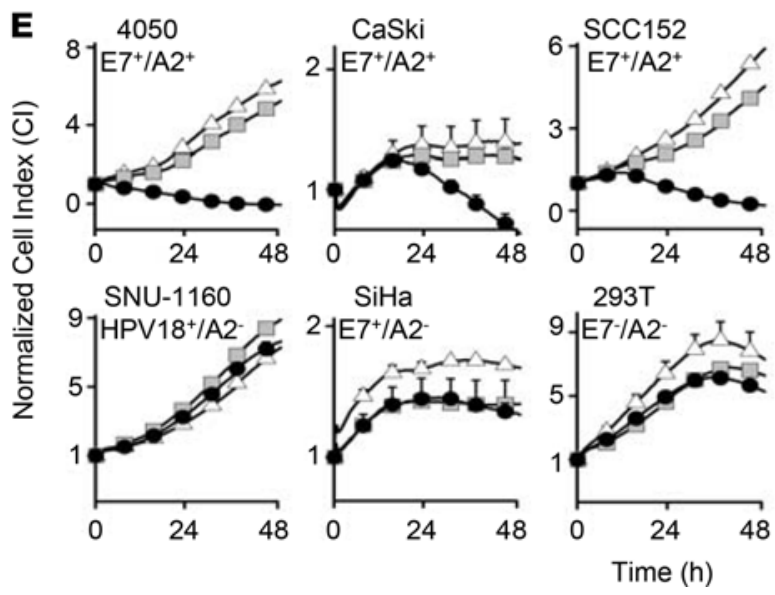
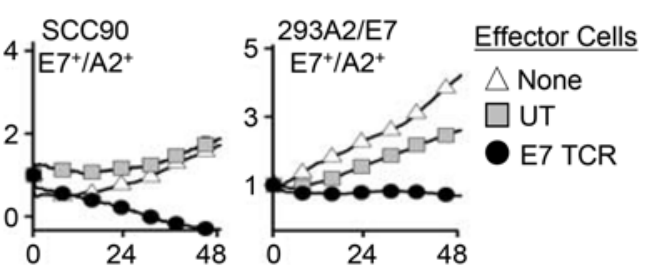

293A2 293A2/E6
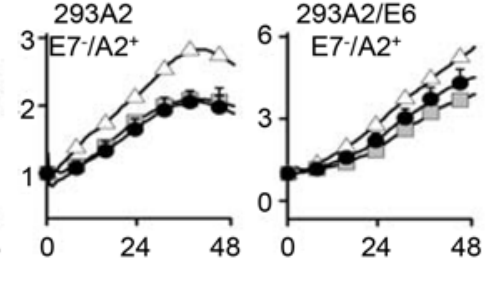

Figure 2. Characterization of the in vitro function of E7 TCR T cells. (A) MHC blocking assay in which the effector cells are E7 TCR T cells and the target cells are as indicated on the $x$ axis. Blocking antibodies against MHC class I or II were added. In the class I control, DMF5 TCR T cells (57) were cocultured with 624 melanoma cells. In the class II control, MAGE A3 TCR T cells (58) were cocultured with 526-CIITA cells. (B) Coculture assay to test for confirmation of the target antigen and restriction element for the E7 TCR. Target cells are 293 cells with or without stable expression of HLA-A*02:01 (293 or 293-A2). They were pulsed with E7 ${ }_{11-19}$ peptide $\left(E 7_{11-19}\right.$ ) or transfected with a plasmid encoding full-length E7 (E7) as indicated on the $x$ axis. OKT3 is a positive control with $\mathrm{T}$ cell stimulation by plate-bound anti-CD3 antibody. (C and $\mathbf{D}$ ) Tumor cell line recognition assays showing the concentration of (C) IFN- $\gamma$ and (D)

TNF- $\alpha$ in supernatants following overnight coculture. 293-A2 cells were pulsed with E7 ${ }_{11-19}$ or E6 ${ }_{29-38}$ peptide as indicated by $x$ axis labels. The other cell lines did not have peptide added. The HPV-16 and HLA-A*02:01 expression of the target cell lines is indicated below each $x$ axis label. (E) E7 TCR T cell-mediated cytolysis of tumor cell lines, as determined by an ACEA xCELLigence Real Time Cell Analyzer. The target cell line name and the expression of HPV-16 E7 and

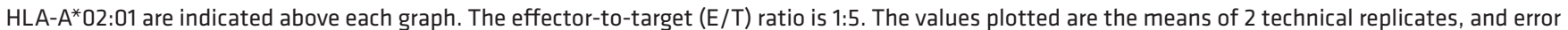
bars represent the SEM. The data displayed are representative of 2 independent experiments. UT, untransduced T cells; E7 TCR, E7 TCR-transduced T cells.

function than the E6 TCR, albeit with some variability depending on the target cells. Greater killing of 4050 cells compared with CaSki cells was a consistent finding that may be related to the greater expression of E7 and HLA-A*02:01 by 4050 (Supplemental Figure 2; supplemental material available online with this article; https://doi.org/10.1172/jci.insight.99488DS1) or to described defects in antigen processing and 
A

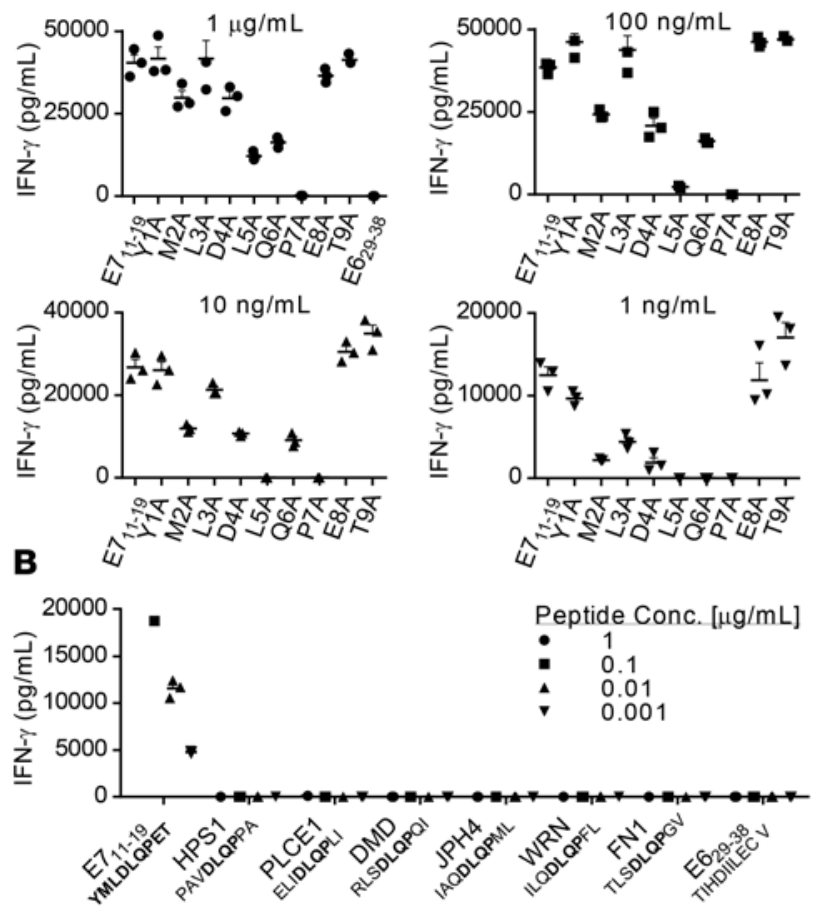

C

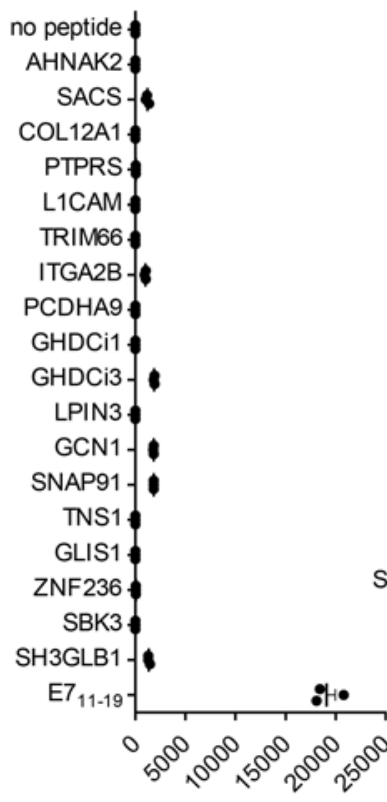

IFN- $\gamma(\mathrm{pg} / \mathrm{mL})$
D

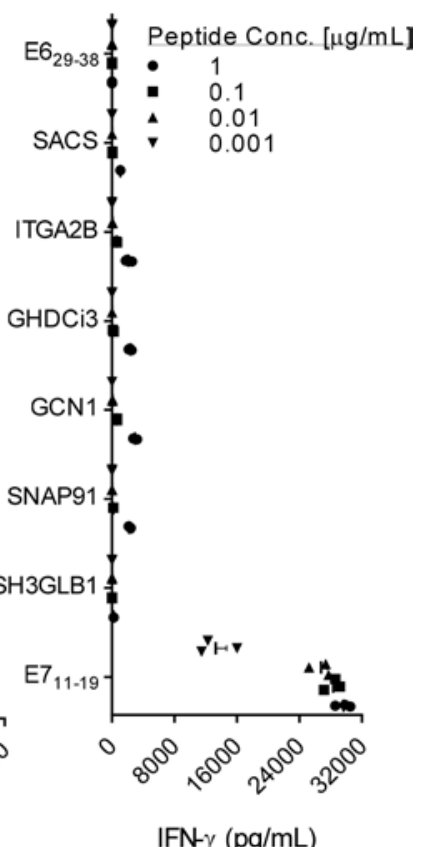

Figure 3. Testing of E7 TCR T cells for cross-reactivity against human peptides. The assays shown were performed by coculture of E7 TCR T cells with T2 cells pulsed with the peptide indicated on the $x$ axis. Coculture supernatants were harvested after $18-24$ hours, and the IFN- $\gamma$ concentration was measured by ELISA. Error bars represent the SEM of 3 technical replicates. (A) To guide cross-reactivity testing, alanine scanning of the E7 ${ }_{11-19}$ epitope was performed. An alanine residue was substituted for the native residue at each position of the E7 ${ }_{11-19}$ (YMLDLQPET) peptide. The residue substitutions are shown on the $x$ axis. The peptide concentration is indicated above each bar graph. (B) Based on the findings in A, the amino acids at positions 4-7 (DLQP) were determined to primarily mediate E7 TCR recognition of $E 7_{11-19}$. Position 2 also affected recognition, presumably due to its role as an $\mathrm{HLA}-\mathrm{A}^{*} 02: 01$ anchor. A BLAST search was conducted to identify human peptides that share these 4 amino acids at the same positions and an HLA-A*02:01 anchor residue at positions 2 ( $L, M, T$, or $A)$ and $9(V, I, L, T$, or $A)(48)$. E7 TCR T cells were tested for reactivity against $T 2$ cells loaded with each of these peptides at the concentration indicated. (C) E7 TCR T cells were tested for recognition of a panel of human peptides that share either 6 residues or 5 residues plus a conservative substitution with $\mathrm{E7}_{11-19}$. Target cells were loaded with $1 \mu \mathrm{g} / \mathrm{ml}$ peptide. (D) E7 TCR T cells were retested for reactivity against peptides identified as possibly reactive in $\mathbf{C}$. The peptide concentrations are indicated.

presentation in the CaSki cell line. They also demonstrated that both TCRs mediated effector functions in CD4 T cells, independent of the CD8 coreceptor, a finding that was consistent with high functional avidity.

E7 TCR T cells induce the regression of HPV16 human cancers in an in vivo model. To investigate the potential for E7 TCR T cells to mediate regression of cancers in vivo, immunodeficient, NOD/SCID $\gamma$ (NSG) mice with established HPV-16 ${ }^{+}$cervical cancer tumors were treated with E7 TCR T cells. A single intravenous injection of E7 TCR T cells was administered either with or without adjuvant intraperitoneal IL-2. E7 TCR $\mathrm{T}$ cells at doses of $1 \times 10^{6}$ or $1 \times 10^{7}$ cells induced complete regression of 4050 tumors. These differences in tumor growth were statistically significant $(P<0.01$ compared with the untreated group or the untransduced T cell group) (Figure 5, A and B). Administration of E7 TCR T cells at a dose of $1 \times 10^{7}$ cells resulted in suppression but not elimination of CaSki tumors. This reduction in tumor growth was also statistically significant $(P<0.05$ compared with the untreated group or the untransduced $\mathrm{T}$ cell group) (Figure $5, \mathrm{C}$ and $\mathrm{D}$ ). Lower doses of E7 TCR T cells $\left(1 \times 10^{5}\right.$ cells per mouse) were not effective against the CaSki tumors. These findings revealed that administration of E7 TCR T cells either alone or in combination with IL-2 could mediate antitumor activity against human cervical cancers in this model.

\section{Discussion}

Here, we describe the discovery of a method to target the constitutively expressed viral oncoprotein, E7, with therapeutic T cells for the treatment of HPV-16-associated epithelial cancers. E7 TCR T cells displayed high avidity for target epitope and no perceptible cross-reactivity against human peptides with similar sequence or TCR-binding residues. In vitro, E7 TCR T cells demonstrated effector T cell 
A

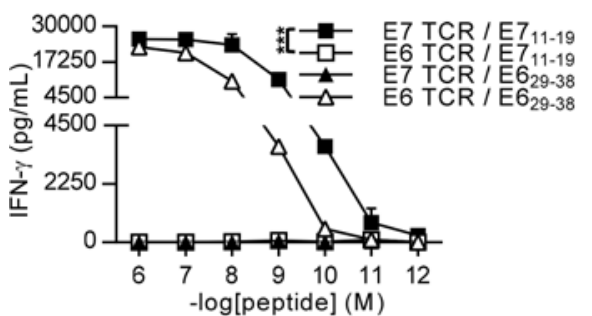

B

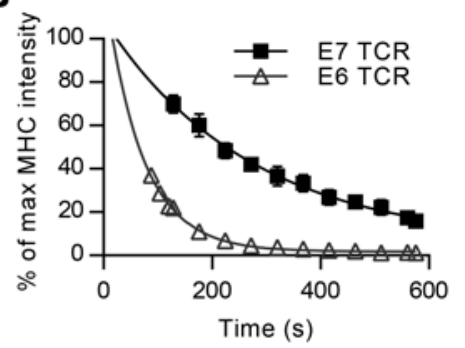

C

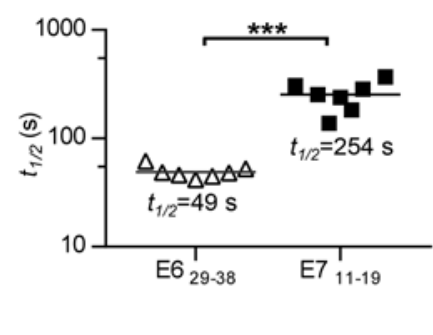

D

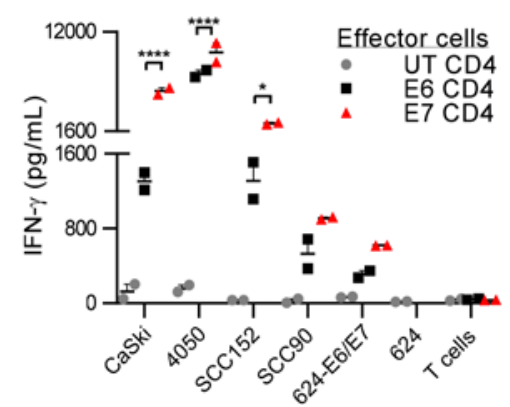

E

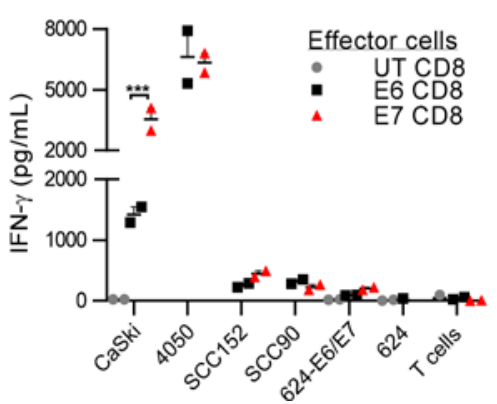

F

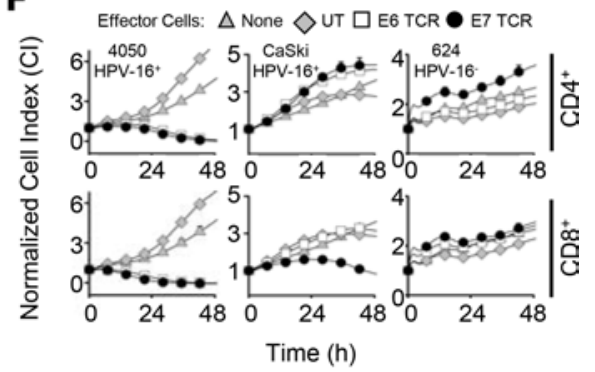

Figure 4. The avidity of E7 TCR T cells for cognate antigen and tumor cell lines. (A) A functional avidity assay is shown with the quantity of IFN- $\gamma$ produced in an overnight coculture graphed. The target cells are T2 cells pulsed with $\mathrm{EG}_{29-38}$ or E7 $_{11-19}$ peptide at the concentrations indicated on the $x$ axis. The TCR/target antigen combinations are shown in the key. Error bars represent the SEM of 3 technical replicates. ${ }^{* *} P<0.001$. (B) $K_{\text {off }}{ }^{*}$ rate assay evaluating the E6 and E7 TCRs. E7 TCR- or E6 TCR-transduced T cells were labeled with reversible, fluorescence-labeled MHC streptamers. The MHC fluorescence intensity of the transduced T cells after the addition of biotin was monitored by flow cytometry. Mean MHC fluorescence intensities of E6 TCR (triangles) and E7 TCR (squares) were extracted for every 8 seconds, normalized, and plotted to calculate the $t_{1 / 2}$ time after fitting an exponential decay curve (7 independent dissociations per TCR, mean with SEM). (C) $t_{1 / 2}$ time from 7 independent peptide-MHC dissociation experiments of the E6 TCR (triangles) and the E7 TCR (squares) are plotted with the median $t_{1 / 2}$ time. ${ }^{* * *} P<0.001$. ( $\mathbf{D}$ and $\mathbf{E}$ ) Cytokine production assay testing the recognition of tumor cell lines by E6 and E7 TCR T cells. (D) CD4 and (E) CD8 T cells were isolated, transduced, and tested separately in functional assays. The quantity of IFN- $\gamma$ produced in an overnight coculture of TCR-transduced T cells and target cells is shown. The target cells are CaSki, 4050, SCC152, SCC90, 624-E6/E7, and 624 cells. Error bars represent the SEM of 2 technical replicates. ${ }^{*} P<0.05,{ }^{* *} P<0.001,{ }^{* * *} P<0.0001$. The result shown is representative of 2 independent experiments. (F) CD4 or CD8 T cells were transduced to express the E6 or E7 TCR and were cocultured with 4050, CaSki, or 624 tumor cell lines. T cell-mediated cytolysis was monitored using the ACEA xCELLigence Real-Time Cell Analyzer. Cell indices plotted in the graphs represent the mean of duplicate samples, and error bars represent the SEM. The result shown is representative of 2 independent experiments. UT, untransduced T cells; E7 TCR, E7 TCR-transduced T cells; E6 TCR, E6 TCR-transduced T cells.

functions, including IFN- $\gamma$ production and tumor cell killing. Multiple head and neck and cervical cancer cells lines were recognized; no cell lines that expressed the targeted peptide-MHC complex were not recognized. In vivo, E7 TCR T cells mediated complete regression of established subcutaneous 4050 tumors and repressed the growth of CaSki tumors in an NSG model. This antitumor activity was observed in the absence of adjuvant therapy (i.e., a vaccine, cytokine, Toll-like receptor agonist, agonistic antibody, or checkpoint inhibitor); at some doses, it was enhanced by the addition of systemic IL-2. The xenograft model in these experiments has limitations, including that it does not recapitulate elements of the human tumor microenvironment, such as the presence of immunosuppressive cells. Another limitation is that the implanted subcutaneous tumors may not reproduce the trafficking requirements for $\mathrm{T}$ cells in humans, which would need to migrate to metastatic tumor sites throughout the patient. Nonetheless, these data provide preclinical support for the now-active first-in-human clinical trial of E7 TCR T cells for HPV-16 ${ }^{+}$epithelial cancers (NCT02858310).

Despite the promise of engineered $\mathrm{T}$ cell therapy in hematological malignancies, the approach has achieved limited progress in epithelial cancers (11). One constraint may be the difficulty in identifying and targeting viable tumor antigens that are highly expressed and isolated to tumor cells (21). The HPV E7 oncoprotein appears to be an ideal target, as it demonstrates (a) high-level, uniform expression by tumors; (b) no expression by vital healthy tissues; (c) functional importance to tumor cells (i.e., promotes tumor cell growth and/or survival); and (d) shared expression between the tumors of different patients. The sequence of E7 varies between HPV types, and the E7 ${ }_{11-19}$ epitope targeted in this study is not shared with other 

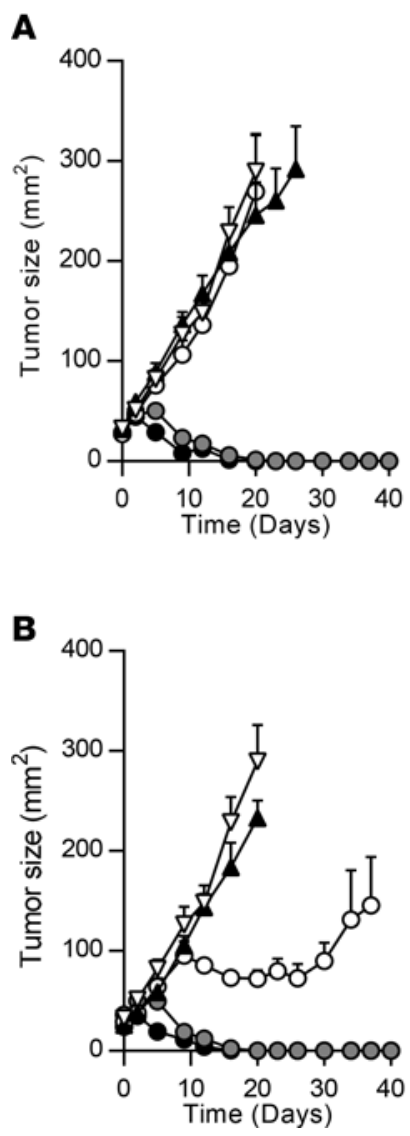

C
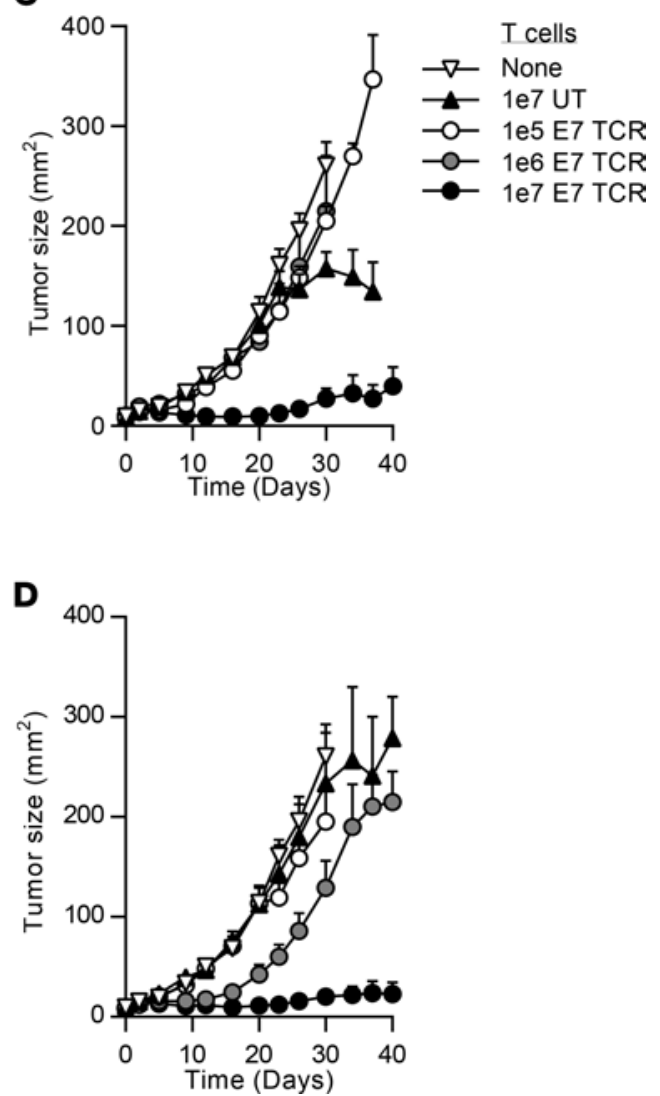

Figure 5. Antitumor activity of E7 TCR T cells against cervical cancer in an in vivo model. NSC mice with 12-day subcutaneous tumors were treated with a single intravenous injection of E7 TCR T cells. The number of T cells is indicated in the key. (A and B) 4050 or (C and D) CaSki human cervical cancer tumors were treated. In B and D, 198,000 IU systemic IL-2 was given daily by intraperitoneal injection for 3 days. The tumor area (product of the longest perpendicular diameters) is plotted on the $y$ axis. Time after $T$ cell injection is plotted on the $x$ axis. The mean values from each group are plotted. Error bars represent the SEM ( $n=5$ mice per group). The results are representative of 2 independent experiments. UT, untransduced T cells; E7 TCR, E7 TCR-transduced T cells.

HPV types, therefore cross-reactivity against tissues that are infected with another HPV type is unlikely. Targeting of an antigen such as E7 may be important for establishing proof of principle for engineered $\mathrm{T}$ cell therapy in an epithelial cancer. In addition, it may help establish a model of adoptive $\mathrm{T}$ cell therapy in human cancer in which toxicity to healthy tissues is not limiting and the mechanisms that control the efficacy of this type of treatment can be studied.

The preclinical assessment of the toxicity of TCR gene-engineered T cells has been challenging (21). Because TCRs target peptide-HLA complexes, and HLA molecules are exclusive to humans, the toxicity of TCR-based treatments can only be studied reliably in humans. Toxicity studies in HLA-transgenic mice are unlikely to be informative because differences in the proteome of mice and humans would greatly confound interpretation. Hence, there is presently no relevant species for toxicity studies in TCR T cell therapy. Instead, toxicity testing primarily consists of evaluation of human tissues for the target antigen and of in vitro probing of the TCR for cross-reactivity against human peptides. E7 is not encoded by the human genome and is expressed only by infected human cells or HPV-induced cancers, which makes its expression by healthy tissues highly unlikely. The theoretical safety of the E7 TCR is also favorable because it is derived from a human and has native complementarity-determining regions (CDRs). Because the TCR was subjected to human thymic selection, it is unlikely to possess high-affinity cross-reactivity against epitopes of human proteins. This contrasts with TCRs of human or murine origin that have been subjected to affinity enhancement through CDR modifications and that have unexpectedly targeted healthy human tissues $(14,20,51)$. To further interrogate the E7 TCR for cross-reactivity, we performed in vitro assays for recognition of human peptides and cross-reactivity was not identified. However, while the theoretical safety profile of E7 TCR T cells is favorable, only a clinical trial in humans can determine its clinical safety.

In summary, we report here for the first time to our knowledge that $\mathrm{T}$ cells can be genetically engineered to target HPV-16 E7 and that these engineered cells can mediate regression of HPV-16 ${ }^{+}$human cancers in an animal model. These findings provided the preclinical basis for an active clinical trial of E7 TCR $\mathrm{T}$ cells for patients with metastatic $\mathrm{HPV}^{+}$cancers. 


\section{Methods}

Tumor cell lines. Cell lines were cultured in RPMI-1640 or DMEM supplemented with 10 percent FBS (MilliporeSigma), GlutaMAX (Life Technologies), $100 \mathrm{U} / \mathrm{ml}$ penicillin (Life Technologies), and $100 \mu \mathrm{g} / \mathrm{ml}$ streptomycin (Life Technologies). CaSki, SiHa, T2, 293, and Panc-1 cell lines were purchased from ATCC. SNU1160 was purchased from the Korean Cell Bank. SCC90 and SCC152 were purchased from the University of Pittsburgh. The 4050 cell line is an HPV- $16^{+}$HLA-A*02:01+ squamous cell cervical cancer line that was generated in our laboratory from a lung metastasis. To generate this cell line, fresh tumor was implanted subcutaneously into NSG mice. Tumors that engrafted and grew were harvested, digested using GentleMACS (Miltenyi Biotec), and grown in IMDM supplemented with $10 \%$ FBS. Tumor cells that expanded in vitro expressed HPV16 E6 and E7, as shown by RT-PCR, displayed similar morphology to other cervical cancer cell lines, and tested negative for mycoplasma. The 3569 TC and 3664 TC cell lines are melanoma cell lines generated previously by the Surgery Branch of the National Cancer Institute. The 624-E6 and 624-E7 cell lines are 624 melanoma cell lines with stable expression of HPV-16 E6 or E7, respectively (38). 293-E7 and 293-A2-E7 cell lines are 293-based cell lines that stably express HPV-16 E7 or HPV-16 E7 and HLA-A*02:01, respectively. They were generated by retroviral transduction with an MSGV2 vector encoding HPV-16 E7 (Gene Oracle). 293-A2 cell lines were generated by the Surgery Branch of the National Cancer Institute (52). The 526-CIITA cell line is a melanoma cell line with stable expression of the class II MHC complex transactivator. All tumor cells lines used in these experiments tested negative for mycoplasma. The identity of the lines was confirmed by HPV-16 E6 and E7 RT-PCR and by morphology and growth characteristics.

HPV E6 and E7 reactivity assay. CILs were tested for HPV-16 oncoprotein reactivity as described previously (36). Briefly, target cells were autologous monocyte-derived dendritic cells loaded with pools of overlapping peptides that spanned E6, E7, or a negative control (gp100) (Miltenyi Biotec). The peptide pools were composed of 15-mers that overlapped by 11 amino acids. Effector cells were CILs that were isolated as described previously (54) and then expanded with a rapid expansion protocol (55). The "E6 Cntrl" expressed a B35-restricted TCR against HPV-16 E6, and the "E7 Cntrl" expressed a DRB1*04-restricted TCR against HPV-16 E7. The readout for reactivity was the concentration of IFN- $\gamma$ in the supernatants following overnight coculture.

TCR isolation and sequencing. 5048 CILs were labeled with PE-conjugated E7 ${ }_{11-19}-\mathrm{HLA}-\mathrm{A}$ *02:01 p-MHC tetramers (NIH Tetramer Core Facility at Emory University). Tetramer-binding cells were selected with anti-PE microbeads (Miltenyi Biotec) and cloned with limited dilution cloning. Following 2 weeks of clonal outgrowth, $\mathrm{T}$ cell clones were screened for tetramer binding by flow cytometry. A clone with strong tetramer binding was identified by flow cytometry; RNA was isolated (Qiagen RNeasy Kit), and cDNA was synthesized (Clontech SMARTer RACE cDNA Amplification Kit). TCR $\alpha$ and $\beta$ chain cDNA were amplified by 5' RACE (Clontech Advantage 2 PCR Kit) with primers specific for the TCR $\alpha$ chain (5'-GCCACAGCACTGTTGCTCTTGAAGTCC-3') and TCR $\beta$ chain (5'-CAGGCAGTATCTGGAGTCATTGAG-3') constant regions. The 5'RACE products were gel purified (Zymo Research) and sequenced (Macrogen).

$T C R$ retroviral constructs and T cell transductions. TCR nucleotide sequences were synthesized and cloned into the MSGV1 retrovirus backbone, which has been used extensively in clinical trials (Gene Oracle). The insert sequence was codon optimized for expression in human tissues. The human TCR constant regions were exchanged for mouse TCR constant regions. For constructs with an additional interchain disulfide bond, cysteine was substituted in place of Thr48 of the $\alpha$ chain and Ser57 of the $\beta$ chain (44). For constructs with hydrophobic substitutions, the $\alpha$ chain transmembrane region sequence was changed from ${ }^{111}$ L $\underline{\text { SVMGLRIL }}{ }^{119}$ to ${ }^{111}$ LLVIVLRIL ${ }^{119}$ (45). A furin P2A linker was used to connect the TCR $\alpha$ and $\beta$ chains in all constructs. The sequence of the fully optimized E7 TCR construct is provided in Supplemental Figure 1.

Human peripheral blood mononuclear cells (PBMCs) were isolated from the buffy coats. Before transduction, PBMCs were cultured in T cell media plus $50 \mathrm{ng} / \mathrm{ml}$ anti-CD3 antibody (OKT3, Miltenyi Biotec) for 2 days. E7 TCR retroviral supernatants were generated by cotransfection of 293GP cells with E7 TCR MSGV1 vector and RD114 plasmid. Two days after transfection, E7 TCR retroviral supernatants were harvested. Transductions were performed on RetroNectin-coated plates (Takara) as described previously (56).

Flow cytometry. Cells were labeled with FITC-, PE-, PE-Cy7-, or APC-H7-conjugated antibodies against CD3, CD4, CD8 (BD Biosciences), or mouse TCR- $\beta$ (clone H5-597, eBioscience). E7 $11-19^{-H L A-A * 02: 01 ~}$ and $\mathrm{E}_{29-38}$-HLA-A*02:01 monomers were provided by the NIH Tetramer Core Facility, and tetramers were assembled and labeled according to the NIH Tetramer Core Facility protocol. Data were acquired with a BD FACSCanto II flow cytometer (BD Biosciences) and analyzed with FlowJo software (FlowJo). 
$T$ cell functional assays. For cytokine production assays, $5 \times 10^{4}$ or $1 \times 10^{5} \mathrm{~T}$ cells were cocultured with an equal number of target cells in 96-well U-bottom plates. For MHC molecule blocking assays, $50 \mu \mathrm{g} / \mathrm{ml}$ anti-MHC I (W6/32, ATCC) or anti-MHC II (HB145, ATCC) antibodies were added to coculture wells, respectively. Reactivity against E6 or E7 HLA-A*02:01-restricted peptides was determined by pulsing T2 or 293-A2 cells with peptide ( $1 \mu \mathrm{M}$, or as indicated in figures) for 2 hours prior to coculture. Coculture supernatants were harvested after 18-24 hours, and IFN- $\gamma$ or TNF- $\alpha$ concentration was measured by ELISA (Thermo Scientific). Cytotoxicity assays were performed by coculture of T cells with target cells at the indicated effector-to-target ratios as described previously (46). Briefly, $1 \times 10^{4}$ target cells per well were seeded and cultured overnight, and then effector $\mathrm{T}$ cells were added at the indicated ratios. Target cell lysis was evaluated with the xCELLigence Real-Time Cell Analyzer (ACEA Biosciences), which assessed electrical impedance due to the adherent cells in each well every 15 minutes until the end of the experiment. The data were processed using the xCELLigence RTCA software package (version 2.0), and the results are reported as a cell index value, which was normalized to 1 at the time when T cells were added. For functional assays comparing the E6 and E7 TCR, constructs for both TCRs used the $\beta-\alpha$ chain order, added constant region, disulfide bond, and the $\alpha$ chain hydrophobic substitutions. Transduction efficiency was equalized between groups by magnetic bead separation based on murine constant region expression.

TCR ligand $K_{\text {off }}$ rate measurement. Up to $5 \times 10^{6} \mathrm{E} 6$ or E7 TCR-transduced healthy donor PBMCs were stained for 45 minutes at $4^{\circ} \mathrm{C}$ with reversible streptamer consisting of Strep-Tactin APC (IBA) and Atto565-labeled HLA-A*02:01/E6 ${ }_{29-38}$ or E7 ${ }_{11-19}$ Strep-tagged monomers in the presence of irreversible BV421-labeled HLA-A*02:01/E6 ${ }_{29-38}$ or E7 ${ }_{11-19}$ MHC multimers in $50 \mu 1$ FACS buffer (PBS supplemented with 0.5\% BSA, EDTA, 0.09\% $\mathrm{NaN}_{3}$ ). Anti-human CD4 AF700 (BD Bioscience) and live/dead stain near IR (Life Technologies) were added during the last 15 minutes before washing and resuspension in $200 \mu \mathrm{l}$ cold FACS buffer. About $1 \times 10^{5}$ stained cells were diluted in $500 \mu 1$ FACS buffer at room temperature and acquired on a BD LSR Fortessa under constant cooling to $20^{\circ} \mathrm{C}$ (Peltier cooler from Qtools). After 30 seconds of acquisition, $500 \mu \mathrm{l}$ of $2 \mathrm{mM}$ biotin were added, and the MHC monomer Atto565 fluorescence intensities of $\mathrm{CD}^{-}$, MHC multimer BV421+', live/dead IR stain ${ }^{-}$lymphocytes were monitored for a total of 10 minutes. Each dissociation was exported as a FCS file for analysis using the same gating strategy to extract the Strep-Tactin APC and MHC monomer Atto565 geometric mean fluorescence intensities (MFIs) every 7-8 seconds with the kinetic tool in FlowJo 10.0 software. Extracted MFI values were plotted against the time points to fit an exponential decay curve into the Strep-Tactin APC MFI values after the addition of biotin (approximately 40-50 seconds after acquisition). The calculated APC dissociation $t_{1 / 2}$ time was used to estimate the starting point for exponential decay fitting into the MHC monomer Atto565 MFI values to calculate TCR ligand $t_{1 / 2}$ times of each dissociation experiment as described previously $(49,50)$.

Treatment of established tumors in a murine model. Animal research protocols were approved by the NIH Animal Care and Use Committee. NSG mice (The Jackson Laboratory) with established human cervical cancer tumors were treated by tail vein injection of human $\mathrm{T}$ cells. Tumors were initiated by subcutaneous injection of $1 \times 10^{6}$ CaSki or 4050 tumor cells on the flank or abdomen. Tumor treatment was on day 12 following tumor cell injection and consisted of a single intravenous injection of T cells (either E7 TCR T cells or untransduced T cells). Some mice received adjuvant IL-2 (aldesleukin, Prometheus Laboratory Inc.) 198,000 IU by intraperitoneal injection daily for 3 days beginning immediately after $\mathrm{T}$ cell injection. Tumor size was determined by caliper measurement of the perpendicular diameters of each tumor and is reported as tumor area.

Statistics. Statistical analyses were performed with Prism 7 (GraphPad Software). A 2-way ANOVA repeated-measures test was used to calculate the statistical significance of the differences in the functional avidity between E7 TCR and E6 TCR. The differences in the production of IFN- $\gamma$ by E7 TCR T cells versus E6 TCR T cells in coculture assays with tumor lines was assessed with 2-way ANOVA multiple comparisons tests. Differences in tumor curves in mouse experiments were determined by 2-way ANOVA repeated-measures multiple comparisons tests. A 2-tailed unpaired $t$ test was used to compare the $K_{\text {off }}$ rate between E7 TCR T cells and E6 TCR T cells. A $P$ value of less than 0.05 was considered significant.

Study approval. Research on archived, deidentified patient samples [CILs and matching PBMCs from patients who had been treated with the pNGLV4A-CRT-E7(detox) vaccine, ref. 53] was approved by the Office of Human Subjects Research, NIH (exemption 11503). 


\section{Author contributions}

$\mathrm{CSH}$ conceived the study, designed experiments, analyzed data, and wrote the manuscript. BYJ conceived the study, designed experiments, performed experiments, analyzed data, and wrote the manuscript. TEC designed experiments, performed experiments, analyzed data, and wrote the manuscript. LMD and BW designed experiments, performed experiments, and analyzed data. SS assisted with experiments and provided feedback. ZY assisted with animal experiments and provided feedback. NPR and SAR provided expertise and feedback. CLT provided patient samples and feedback.

\section{Acknowledgments}

This research was supported by the Intramural Research Program of the NIH and by cooperative research and development agreements with Kite Pharma.

Address correspondence to: Christian S. Hinrichs, National Cancer Institute - Experimental Transplantation and Immunology Branch, NIH, 10 Center Drive, Room 4B04, Bethesda, Maryland 20892, USA. Phone: 301.435.3027; Email: hinrichs@mail.nih.gov.

1. zur Hausen H. Papillomaviruses and cancer: from basic studies to clinical application. Nat Rev Cancer. 2002;2(5):342-350

2. Maxwell JH, Grandis JR, Ferris RL. HPV-associated head and neck cancer: unique features of epidemiology and clinical management. Annu Rev Med. 2016;67:91-101.

3. Moody CA, Laimins LA. Human papillomavirus oncoproteins: pathways to transformation. Nat Rev Cancer. 2010;10(8):550-560.

4. Ferris RL, et al. Nivolumab for recurrent squamous-cell carcinoma of the head and neck. $N$ Engl J Med. 2016;375(19):1856-1867.

5. Tewari KS. Clinical implications for cediranib in advanced cervical cancer. Lancet Oncol. 2015;16(15):1447-1448.

6. Vermorken JB, et al. Platinum-based chemotherapy plus cetuximab in head and neck cancer. $N$ Engl J Med. 2008;359(11):1116-1127.

7. Siegel RL, Miller KD, Jemal A. Cancer statistics, 2017. CA Cancer J Clin. 2017;67(1):7-30.

8. Torre LA, Siegel RL, Ward EM, Jemal A. Global cancer incidence and mortality rates and trends--an update. Cancer Epidemiol Biomarkers Prev. 2016;25(1):16-27.

9. Hinrichs CS, Rosenberg SA. Exploiting the curative potential of adoptive T-cell therapy for cancer. Immunol Rev. 2014;257(1):56-71.

10. Lizée G, Overwijk WW, Radvanyi L, Gao J, Sharma P, Hwu P. Harnessing the power of the immune system to target cancer. Annu Rev Med. 2013;64:71-90.

11. Hinrichs CS. Molecular pathways: breaking the epithelial cancer barrier for chimeric antigen receptor and T-cell receptor gene therapy. Clin Cancer Res. 2016;22(7):1559-1564.

12. Schmitt TM, Stromnes IM, Chapuis AG, Greenberg PD. New strategies in engineering T-cell receptor gene-modified t cells to more effectively target malignancies. Clin Cancer Res. 2015;21(23):5191-5197.

13. Brentjens RJ, et al. CD19-targeted T cells rapidly induce molecular remissions in adults with chemotherapy-refractory acute lymphoblastic leukemia. Sci Transl Med. 2013;5(177):177ra38.

14. Grupp SA, et al. Chimeric antigen receptor-modified T cells for acute lymphoid leukemia. NEngl J Med. 2013;368(16):1509-1518.

15. Porter DL, et al. Chimeric antigen receptor T cells persist and induce sustained remissions in relapsed refractory chronic lymphocytic leukemia. Sci Transl Med. 2015;7(303):303ra139.

16. Kochenderfer JN, et al. Chemotherapy-refractory diffuse large B-cell lymphoma and indolent B-cell malignancies can be effectively treated with autologous T cells expressing an anti-CD19 chimeric antigen receptor. J Clin Oncol. 2015;33(6):540-549.

17. Robbins PF, et al. A pilot trial using lymphocytes genetically engineered with an NY-ESO-1-reactive T-cell receptor: long-term follow-up and correlates with response. Clin Cancer Res. 2015;21(5):1019-1027.

18. Linette GP, et al. Cardiovascular toxicity and titin cross-reactivity of affinity-enhanced T cells in myeloma and melanoma. Blood. 2013;122(6):863-871.

19. Parkhurst MR, et al. T cells targeting carcinoembryonic antigen can mediate regression of metastatic colorectal cancer but induce severe transient colitis. Mol Ther. 2011;19(3):620-626.

20. Morgan RA, et al. Cancer regression and neurological toxicity following anti-MAGE-A3 TCR gene therapy. J Immunother. 2013;36(2):133-151.

21. Hinrichs CS, Restifo NP. Reassessing target antigens for adoptive T-cell therapy. Nat Biotechnol. 2013;31(11):999-1008.

22. Hellner K, Münger K. Human papillomaviruses as therapeutic targets in human cancer. J Clin Oncol. 2011;29(13):1785-1794.

23. Lee SJ, Yang A, Wu TC, Hung CF. Immunotherapy for human papillomavirus-associated disease and cervical cancer: review of clinical and translational research. J Gynecol Oncol. 2016;27(5):e51.

24. Natale C, Giannini T, Lucchese A, Kanduc D. Computer-assisted analysis of molecular mimicry between human papillomavirus 16 E7 oncoprotein and human protein sequences. Immunol Cell Biol. 2000;78(6):580-585.

25. Crosbie EJ, Einstein MH, Franceschi S, Kitchener HC. Human papillomavirus and cervical cancer. Lancet. 2013;382(9895):889-899.

26. Fernandes JV, et al. Link between chronic inflammation and human papillomavirus-induced carcinogenesis (Review). Oncol Lett. 2015;9(3):1015-1026.

27. Piersma SJ. Immunosuppressive tumor microenvironment in cervical cancer patients. Cancer Microenviron. 2011;4(3):361-375.

28. Evans M, et al. Antigen processing defects in cervical carcinomas limit the presentation of a CTL epitope from human papillo- 
mavirus 16 E6. J Immunol. 2001;167(9):5420-5428.

29. Westrich JA, Warren CJ, Pyeon D. Evasion of host immune defenses by human papillomavirus. Virus Res. 2017;231:21-33.

30. Höhn H, et al. CD4+ tumor-infiltrating lymphocytes in cervical cancer recognize HLA-DR-restricted peptides provided by human papillomavirus-E7. J Immunol. 1999;163(10):5715-5722.

31. Ghosh AK, Moore M. Tumour-infiltrating lymphocytes in cervical carcinoma. Eur J Cancer. 1992;28A(11):1910-1916.

32. van Meir H, et al. The need for improvement of the treatment of advanced and metastatic cervical cancer, the rationale for combined chemo-immunotherapy. Anticancer Agents Med Chem. 2014;14(2):190-203.

33. Tewari KS, Monk BJ. New strategies in advanced cervical cancer: from angiogenesis blockade to immunotherapy. Clin Cancer Res. 2014;20(21):5349-5358.

34. Sun YY, et al. Local HPV recombinant vaccinia boost following priming with an HPV DNA vaccine enhances local HPV-specific CD8+ T-cell-mediated tumor control in the genital tract. Clin Cancer Res. 2016;22(3):657-669.

35. Kenter GG, et al. Phase I immunotherapeutic trial with long peptides spanning the E6 and E7 sequences of high-risk human papillomavirus 16 in end-stage cervical cancer patients shows low toxicity and robust immunogenicity. Clin Cancer Res. 2008;14(1):169-177.

36. Stevanović S, et al. Complete regression of metastatic cervical cancer after treatment with human papillomavirus-targeted tumor-infiltrating T cells. J Clin Oncol. 2015;33(14):1543-1550.

37. Stevanović S, et al. Landscape of immunogenic tumor antigens in successful immunotherapy of virally induced epithelial cancer. Science. 2017;356(6334):200-205

38. Draper LM, et al. Targeting of HPV-16+ epithelial cancer cells by TCR gene engineered T cells directed against E6. Clin Cancer Res. 2015;21(19):4431-4439.

39. Gillison ML, Chaturvedi AK, Lowy DR. HPV prophylactic vaccines and the potential prevention of noncervical cancers in both men and women. Cancer. 2008;113(10 Suppl):3036-3046.

40. Gonzalez-Galarza FF, Christmas S, Middleton D, Jones AR. Allele frequency net: a database and online repository for immune gene frequencies in worldwide populations. Nucleic Acids Res. 2011;39(Database issue):D913-D919.

41. Riemer AB, et al. A conserved E7-derived cytotoxic T lymphocyte epitope expressed on human papillomavirus 16-transformed HLA-A2+ epithelial cancers. J Biol Chem. 2010;285(38):29608-29622.

42. Davis JL, Theoret MR, Zheng Z, Lamers CH, Rosenberg SA, Morgan RA. Development of human anti-murine T-cell receptor antibodies in both responding and nonresponding patients enrolled in TCR gene therapy trials. Clin Cancer Res. 2010;16(23):5852-5861.

43. Cohen CJ, Zhao Y, Zheng Z, Rosenberg SA, Morgan RA. Enhanced antitumor activity of murine-human hybrid T-cell receptor (TCR) in human lymphocytes is associated with improved pairing and TCR/CD3 stability. Cancer Res. 2006;66(17):8878-8886.

44. Cohen CJ, Li YF, El-Gamil M, Robbins PF, Rosenberg SA, Morgan RA. Enhanced antitumor activity of T cells engineered to express T-cell receptors with a second disulfide bond. Cancer Res. 2007;67(8):3898-3903.

45. Haga-Friedman A, Horovitz-Fried M, Cohen CJ. Incorporation of transmembrane hydrophobic mutations in the TCR enhance its surface expression and T cell functional avidity. J Immunol. 2012;188(11):5538-5546.

46. Peper JK, Schuster H, Löffler MW, Schmid-Horch B, Rammensee HG, Stevanović S. An impedance-based cytotoxicity assay for real-time and label-free assessment of T-cell-mediated killing of adherent cells. J Immunol Methods. 2014;405:192-198.

47. Cameron BJ, et al. Identification of a Titin-derived HLA-A1-presented peptide as a cross-reactive target for engineered MAGE A3-directed T cells. Sci Transl Med. 2013;5(197):197ra103

48. Kast WM, et al. Role of HLA-A motifs in identification of potential CTL epitopes in human papillomavirus type 16 E6 and E7 proteins. J Immunol. 1994;152(8):3904-3912.

49. Nauerth M, et al. TCR-ligand koff rate correlates with the protective capacity of antigen-specific CD8+ T cells for adoptive transfer. Sci Transl Med. 2013;5(192):192ra87.

50. Nauerth M, et al. Flow cytometry-based TCR-ligand Koff-rate assay for fast avidity screening of even very small antigen-specific T cell populations ex vivo. Cytometry A. 2016;89(9):816-825.

51. Porter DL, Levine BL, Kalos M, Bagg A, June CH. Chimeric antigen receptor-modified T cells in chronic lymphoid leukemia NEngl J Med. 2011;365(8):725-733.

52. Khong HT, Rosenberg SA. Pre-existing immunity to tyrosinase-related protein (TRP)-2, a new TRP-2 isoform, and the NY-ESO-1 melanoma antigen in a patient with a dramatic response to immunotherapy. J Immunol. 2002;168(2):951-956.

53. Alvarez RD, et al. A pilot study of pNGVL4a-CRT/E7(detox) for the treatment of patients with HPV16+ cervical intraepithelial neoplasia 2/3 (CIN2/3). Gynecol Oncol. 2016;140(2):245-252.

54. Clark RA, et al. A novel method for the isolation of skin resident T cells from normal and diseased human skin. J Invest Dermatol. 2006;126(5):1059-1070.

55. Dudley ME, Wunderlich JR, Shelton TE, Even J, Rosenberg SA. Generation of tumor-infiltrating lymphocyte cultures for use in adoptive transfer therapy for melanoma patients. J Immunother. 2003;26(4):332-342.

56. Hinrichs CS, et al. Human effector CD8+ T cells derived from naive rather than memory subsets possess superior traits for adoptive immunotherapy. Blood. 2011;117(3):808-814.

57. Johnson LA, et al. Gene therapy with human and mouse T-cell receptors mediates cancer regression and targets normal tissues expressing cognate antigen. Blood. 2009;114(3):535-546.

58. Yao X, et al. Isolation and characterization of an HLA-DPB1*04: 01-restricted MAGE-A3 T-cell receptor for cancer immunotherapy. J Immunother. 2016;39(5):191-201. 